\title{
Solubility and Toxicity Level of Monascus Pigments
}

\author{
Anna Yuliana ${ }^{1,2 *}$, Marlia Singgih Wibowo ${ }^{1}$ and Elin Julianti ${ }^{1}$
}

${ }^{1}$ School of Pharmacy, Bandung Institute of Technology, Indonesia

${ }^{2}$ Departement of Pharmacy, STIKes Bakti Tunas Husada, Indonesia

Submission: June 13, 2018; Published: July 23, 2018

*Corresponding author: Anna Yuliana, School of Pharmacy, Bandung Institute of Technology, Ganesa 10 Bandung, West Java, Indonesia; Tel.: +6281222903376; Email: anna_yuliana@stikes-bth.ac.id

\begin{abstract}
Research on Monascus pigments (MPs) has been progressing very rapidly. The progresses include the discovery of new pigments and methods used for isolating and identifying the new pigments. Currently, nearly fifty-seven new pigments have been found as derivations of the six main pigments existed. The process of new pigments discovery is unable to separate from that of fermentation or isolation process. In several new pigments, there was no complete information relating to solubility and level of toxicity. This research covers data of all fifty-seven new pigments including color, molecular weight, water solubility, log Kow (octanol-water partition coefficient), and baseline toxicity level by Ecological Structure Activity Relationships (ECOSAR) Program. These results are expected to give more comprehensive data about Monascus pigments.
\end{abstract}

Keywords: Monascus pigments; Solubility; Toxicity; Ecosar

\section{Introduction}

Monascus Pigments (MPs) has long been used as a natural food colorant, especially in some Asian countries such as South China. Monascus produces red yeast rice, which is rice covered with a red Monascus sp. Angkak can be used as a dye for yoghurt, bacon, and sausage and also for the preservative of fruits, vegetables, and fish products [1].

The Ecological Structure Activity Relationships (ECOSAR) is a computerized predictive system that estimates aquatic toxicity. The program estimates a chemical's acute (short-term) Result

Table 1: Solubility and Toxicity Level of Monascus Pigments.

\begin{tabular}{|c|c|c|c|c|c|c|c|c|}
\hline \multirow{2}{*}{ No } & \multirow{2}{*}{ Pigments } & \multirow{2}{*}{ Color } & \multirow{2}{*}{$\begin{array}{l}\text { MW (g/ } \\
\text { mol) }\end{array}$} & \multirow{2}{*}{$\begin{array}{c}\text { Water } \\
\text { Solubility } \\
\text { (mg/L) }\end{array}$} & \multirow{2}{*}{ Log Kow } & \multicolumn{3}{|c|}{$\begin{array}{c}\text { Neutral organic SAR (Baseline } \\
\text { Toxicity) }(\mathrm{mg} / \mathrm{L})\end{array}$} \\
\hline & & & & & & $\begin{array}{c}\text { Fish LC } \\
96 \mathrm{~h} \\
\end{array}$ & $\begin{array}{l}\text { Daphnid } \\
\text { LC }_{50}, 48 \mathrm{~h}\end{array}$ & $\begin{array}{l}\text { Green Algae } \\
\mathrm{EC}_{50}, 96 \mathrm{~h}\end{array}$ \\
\hline 1 & Glycyl Rubropuctamin & Red & 413.18 & 18.68 & 0.071 & 18328.39 & 6725.24 & 6168.59 \\
\hline 2 & Isolate from MPs 1 & Red & 510.25 & 4.793 & 0.043 & 24022.46 & 8766.53 & 8039.59 \\
\hline 3 & Isolate from MPs 2 & Red & 538.28 & 5.417 & 2.2 & 292.25 & 160.59 & 149.11 \\
\hline 4 & Isolate from MPs 3 & Red & 439.20 & 0.6096 & 1.621 & 790.75 & 389.32 & 360.29 \\
\hline 5 & Isolate from MPs 4 & Red & 439.24 & 0.2773 & 2.021 & 345.60 & 183.58 & 170.28 \\
\hline 6 & N-glutaryl Monascorubramine & Red & 511.22 & 0.4876 & 1.197 & 2209.66 & 1003.9 & 926.80 \\
\hline 7 & N-glutaril Rubropuctamine & Red & 483.19 & 5.089 & 0.215 & 15920.09 & 6003.13 & 5510.82 \\
\hline 8 & N-glucosyl rubropunctamine & Red & 557.64 & 0.2901 & 3.536 & 19.1 & 13.53 & 12.66 \\
\hline 9 & N-glucosyl monascorubramine & Red & 585.69 & 0.02759 & 4.519 & 2.63 & 2.25 & 2.11 \\
\hline 10 & Compound R3 & Red & 374.17 & 336.5 & 1.905 & 374.68 & 194.67 & 180.45 \\
\hline
\end{tabular}

toxicity and chronic (long-term or delayed) toxicity to aquatic organisms, such as fish, aquatic invertebrates, and aquatic plants, by using computerized Structure Activity Relationships (SARs) [2].

\section{Materials and Methods}

Data collected from various studies that have been done before [3-5] and added with some of the data from ECOSAR results. This toxicity test on ECOSAR program was used to determine the toxicity against water organisms. 
Trends in Technical \& Scientific Research

\begin{tabular}{|c|c|c|c|c|c|c|c|c|}
\hline 11 & Red Derivative 1 & Red & 453.22 & 0.2188 & 2.039 & 344.06 & 183.36 & 170.1 \\
\hline 12 & Red Derivative 2 & Red & 425.18 & 2.266 & 1.056 & 2460.49 & 1088.33 & 1003.93 \\
\hline 13 & Red Derivative 3 & Red & 497.20 & 1.576 & 0.706 & 5933.47 & 2455.88 & 2260.86 \\
\hline 14 & Red Derivative 4 & Red & 437.54 & 0.5376 & 4.586 & 1.71 & 1.48 & 1.39 \\
\hline 15 & Red Derivative 5 & Red & 453.22 & 0.2188 & 2.039 & 344.06 & 183.36 & 170.1 \\
\hline 16 & Red Derivative 6 & Red & 425.18 & 2.266 & 1.056 & 2460.49 & 1088.33 & 1003.93 \\
\hline 17 & Red Derivative 7 & Red & 497.20 & 1.576 & 0.706 & 5933.47 & 2455.88 & 2260.86 \\
\hline 18 & Red Derivative 8 & Red & 469.17 & 16.42 & -0.276 & 42679.29 & 14661.65 & 13421.26 \\
\hline 19 & Un Named & Red & 375.20 & 1636 & 1.676 & 602.53 & 299.78 & 277.52 \\
\hline 20 & Manascopyridine A & Red & 355.18 & 231.1 & 1.634 & 622.53 & 307.25 & 284.37 \\
\hline 21 & Manascopyridine B & Red & 383.21 & 22.58 & 2.616 & 88.11 & 52.40 & 48.77 \\
\hline 22 & Manascopyridine C & Red & 357.19 & 795.3 & 1.588 & 687.97 & 336.62 & 311.47 \\
\hline 23 & Monascopyridine D & Red & 343.21 & 25.02 & 3.446 & 14.17 & 9.87 & 9.23 \\
\hline 24 & New red pigment & Red & 375.20 & 1636 & 1.676 & 602.53 & 299.78 & 277.52 \\
\hline 25 & Red Shandong 1 & Red & 303.40 & 1973 & 2.087 & 208.25 & 112.01 & 103.93 \\
\hline 26 & Red Shandong 2 & Red & 331.46 & 195.2 & 3.069 & 29.85 & 19.34 & 18.05 \\
\hline 27 & PP-V & Purple red & 411.17 & 2612 & 0.46 & 8164.14 & 3224.95 & 2964.64 \\
\hline 28 & Monaphilol A & Orange & 384.19 & 11.23 & 3.562 & 12.51 & 8.90 & 8.33 \\
\hline 29 & Monaphilol B & Orange & 356.16 & 114.9 & 2.579 & 88.37 & 52.18 & 48.56 \\
\hline 30 & Monaphilol C & Orange & 440.22 & 16.81 & 2.947 & 51.01 & 32.3 & 30.12 \\
\hline 31 & Monaphilol D & Orange & 412.48 & 173.8 & 1.965 & 364.07 & 191.34 & 177.43 \\
\hline 32 & Monascusone A & Yellow & 254.12 & 2608 & 1.094 & 1361.60 & 606.53 & 559.61 \\
\hline 33 & Monascusone B & Yellow & 302.12 & 1972 & 0.914 & 2346.48 & 1010.26 & 931.14 \\
\hline 34 & Xanthomonascin A & Yellow & 388.15 & 383.3 & 1.738 & 548.76 & 276.24 & 255.81 \\
\hline 35 & Xanthomonascin B & Yellow & 414.20 & 18.8 & 3.082 & 36.36 & 23.62 & 22.04 \\
\hline 36 & Yellow II & Yellow & 372.19 & 6.886 & 3.299 & 20.84 & 14.1 & 13.18 \\
\hline 37 & FK17-P2B2 & Yellow & 236.10 & 242.9 & 2.417 & 81.98 & 46.94 & 43.64 \\
\hline 38 & Monaphilones A & Yellow & 360.49 & 5.540 & 4.192 & 3.06 & 2.45 & 2.31 \\
\hline 39 & Monaphilones B & Yellow & 332.20 & 56.21 & 3.21 & 21.44 & 14.27 & 13.33 \\
\hline 40 & Monaphilones C & Yellow & 336.23 & 97.28 & 2.903 & 40.95 & 25.71 & 23.97 \\
\hline 41 & Y3 & Yellow & 448.21 & 897.6 & 0.731 & 5085.16 & 2114.57 & 1946.93 \\
\hline 42 & Monashexenone (Y) & Yellow & 320.20 & 86.53 & 2.976 & 34.95 & 22.25 & 20.76 \\
\hline 43 & Rubropunctin & Yellow & 358.21 & 0.3022 & 5.486 & 0.23 & 0.23 & 0.22 \\
\hline 44 & Monarubrin $(\mathrm{Y}, \mathrm{BF})$ & Yellow & 330.18 & 29.06 & 3.559 & 10.34 & 7.35 & 6.88 \\
\hline 45 & Purpureusone & Yellow & 390.24 & 12.92 & 2.849 & 55.40 & 34.43 & 32.09 \\
\hline 46 & Monapurones A & Yellow oil & 330.18 & 3.322 & 4.466 & 1.73 & 1.46 & 1.37 \\
\hline 47 & Monapurones B & Yellow oil & 344.20 & 0.02141 & 6.434 & 0.03 & 0.04 & 0.03 \\
\hline 48 & Monapurones C & Yellow oil & 344.20 & 0.02141 & 6.434 & 0.03 & 0.04 & 0.03 \\
\hline 49 & Monascuspiloin & Yellowish oil & 360.45 & 22.07 & 3.39 & 16.72 & 11.52 & 10.77 \\
\hline 50 & Monankarin A-B & $\begin{array}{l}\text { Yellow } \\
\text { needles }\end{array}$ & 358.14 & 2754 & 1.629 & 633.97 & 312.61 & 289.32 \\
\hline 51 & Monankarin C-D & $\begin{array}{c}\text { Yellow } \\
\text { needles }\end{array}$ & 372.16 & 771.2 & 2.176 & 212.43 & 116.21 & 107.89 \\
\hline 52 & Monankarin E & $\begin{array}{l}\text { Yellow } \\
\text { needles }\end{array}$ & 344.13 & 7610 & 1.212 & 1444.69 & 658.11 & 607.62 \\
\hline 53 & Monankarin F & $\begin{array}{c}\text { Yellow } \\
\text { needles }\end{array}$ & 356.16 & 80.15 & 3.442 & 14.85 & 10.33 & 9.66 \\
\hline
\end{tabular}




\section{Trends in Technical \& Scientific Research}

\begin{tabular}{|c|c|c|c|c|c|c|c|c|}
\hline 54 & Monasfluor A & $\begin{array}{c}\text { Blue } \\
\text { fluorescent }\end{array}$ & 354.18 & 0.08076 & 5.688 & 0.14 & 0.15 & 0.14 \\
\hline 55 & Monasfluor B & $\begin{array}{c}\text { Blue } \\
\text { fluorescent }\end{array}$ & 384.19 & 0.9693 & 4.21 & 3.27 & 2.63 & 2.47 \\
\hline 56 & Monascuskaodione A & Colorless oil & 356.42 & 9.922 & 3.228 & 23.11 & 15.43 & 14.42 \\
\hline 57 & Monascuskaodione B & Colorles oil & 384.47 & 4.210 & 0,969 & 3.27 & 2.63 & 2.47 \\
\hline
\end{tabular}

As shown in Table 1, data showed that the lower log Kow of a compound, the lower of toxicity. And from the toxicity point of view, those of compounds to categorize having lower toxicity than others were LC50 and EC50 values $>100 \mathrm{mg} / \mathrm{L}$. This was because the lower binding energy of a compound, the stronger bond between the receptor and that compound. It made the compound attach to biological membranes longer and more toxicable [2].

\section{Conclusion}

Based on log Kow value of fifty-seven pigments of Monascus were determined, Red Pigment 8 has the lowest toxicity while Monapurones (B and C) have the highest toxicity.

\section{References}

1. Singgih W, Dan Julianti E (2015) Food colorant from microorganisms. In: Liong MT (Edt.) Beneficial Microorganism in Food and Nutraceuticals, Microbiology Monographs 27.

2. Benfenati E, Gini G, Piclin N, Roncaglioni A, Vari MR (2003) Predicting $\operatorname{logP}$ of pesticides using different software. Chemosphere 53(9): 11551164.

3. Feng Y, Shao Y, Chen F (2012) Monascus pigments. Appl Microbiol Biotechnol 96(6): 1421-1440.

4. Patakova P (2013) Monascus secondary metabolites: production and biolitical activity. J Ind Microbiol Biotecnol 40(2): 169-181.

5. Mostafa ME, Abbady MS (2014) Secondary metabolites and Bioactivity of the Monascus pigments review article. Global Journal of Biotechnology \& Biochemistry 9(1): 1-13.
This work is licensed under Creative Commons Attribution 4.0 License DOI: 10.19080/TTSR.2018.02.555594
Your next submission with Juniper Publishers will reach you the below assets

- Quality Editorial service

- Swift Peer Review

- Reprints availability

- E-prints Service

- Manuscript Podcast for convenient understanding

- Global attainment for your research

- Manuscript accessibility in different formats

( Pdf, E-pub, Full Text, Audio)

- Unceasing customer service

Track the below URL for one-step submission https://juniperpublishers.com/online-submission.php 